\title{
EFFECT OF DUAL-TRACK INTERACTIVE NURSING INTERVENTION MODEL ON ANXIETY AND DEPRESSION IN PATIENTS WITH CORONARY HEART DISEASE
}

\author{
Jiao Yang ${ }^{1}$, Hongling $\mathrm{Hu}^{2} \&$ Yamin $\mathrm{Li}^{1}$ \\ ${ }^{I}$ The Second Xiangya Hospital, Central South University, Changsha, Hunan, China \\ ${ }^{2}$ Xiangya Hospital, Central South University, Changsha, Hunan, China
}

received: 26.2.2020;

revised: 15.4.2020;

accepted: 5.5 .2020

\section{SUMMARY}

Background: Elderly patients with coronary heart disease often suffer adverse psychological reactions, such as anxiety and depression. The dual-track interactive nursing model is a nursing intervention aimed to provide specific and community nursing. For patients with chronic diseases, this model can improve the patients' self-management and rehabilitation. The effect of this model on the mental health of patients with chronic diseases has been unanimously recognized by researchers. In this study, a dual-track interactive nursing model intervention was conducted on the anxiety and depression in elderly patients with coronary heart disease to verify the psychological effect of the model.

Subjects and methods: From June 2018 to June 2019, 136 elderly patients with coronary heart disease (mean age of $63.5 \pm 8.26$ years) from three communities in Changsha, Hunan Province, China were selected as subjects. A total of 53 and 50 patients were identified in the intervention and the control groups, respectively. The control group underwent routine longitudinal referral, whereas the intervention group was subjected to a two-track interactive nursing model intervention. The Short Form-36 Health Survey (SF-36) and related questionnaires were used to monitor and compare the two groups before and after the intervention and employed for scoring and comparative analysis.

Results: After the intervention, the mental health scores of the intervention group in total score, somatization, obsessive-compulsive symptoms, interpersonal sensitivity, depression, anxiety, hostility, and paranoia are significantly lower than those of the control group $(P<0.05)$. The intervention group has scored significantly higher in coping style but significantly lower in negative coping than the control group $(P<0.05)$.

Conclusions: The application of the dual-track interactive nursing model intervention in the management of patients with coronary heart disease can improve the self-management and the mental health of patients with coronary heart disease.

Key words: dual-track interaction - intervention model - coronary heart disease - anxiety - depression

\section{INTRODUCTION}

Chronic diseases are one of the common diseases and causes of death among the elderly (Vermunt et al. 2017). Based on the "China Report of the Development on Aging Cause (2013)", China has entered an accelerated period of aging as the elderly population exceeded 200 million in 2013. Methods to improve the health of the elderly is an important guarantee for practicing healthy aging. Studies have shown that the prevalence of chronic diseases among Chinese people over the age of 60 is more than three times than that of the general population and higher than that at the international level. Chronic diseases have become an important factor influencing the physical and mental health of the Chinese elderly (Yin et al. 2016). Among various chronic diseases, coronary heart disease (CHD), a type of cardiovascular disease with a very high fatality rate, is the leading cause of death among the elderly (Zhang et al. 2018). As revealed in a survey, $60 \%$ of the total elderly population in the United States died from CHD (Valbusa et al. 2018). In addition, based on relevant statistics in China, the incidence and fatality rates of CHD increase every year, roughly equivalent in rural and urban areas. Moreover, the fatality rate is higher among the male population. CHD includes angina pectoris and myocardial infarction. Although not contagious, CHD is featured by high incidence, unpredictable changes, high incoming speed, and high mortality (Wagner et al. 2018). CHD threatens the overall health level, increases the medical expenses, and lowers the living standards of the elderly (Catalan-Serra et al. 2019). Moreover, CHD brings high psychological pressure to the elderly, resulting in negative emotions, such as anxiety and depression, which affect the subjective wellbeing and quality of life of the elderly. Among these psychological problems, anxiety and depression are common problems in elderly patients with CHD. If these negative emotions are not effectively controlled, the condition of CHD in elderly patients may aggravate. The aggravation of the disease will, in turn, lead to the generation of more negative emotions, forming a vicious closed loop. In a previous study of 367703 European people, Khandaker et al. (2019) revealed that the comorbidity between CHD and depression is largely caused by common environmental factors. Furthermore, depression and anxiety are important factors affecting the prognosis of CHD. Positive emotions are conducive to the rapid recovery of patients compared with the emotional state of negative depression. 
Strengthening prognostic emotional nursing of patients with CHD is of importance due to the threats brought by psychological problems, such as depression and anxiety, to elderly patients with CHD. The most prevalent and common intervention method is the cognitive-behavioral intervention technology, which includes health promotion, diet, and exercise guidance. This method is simple and easy to operate, but the effect is not satisfactory (Richards et al. 2018). Physical exercise is also a form of intervention and has a remarkableb effect in preventing and relieving depression in the general population and in groups with CHD (Schuch et al. 2015). However, a single intervention method generally fails to produce a long-term intervention effect. Therefore, the dual-track interactive nursing intervention model has gradually attracted the attention of researchers. Although certain researchers have explored the application of this model in the treatment of diabetes and other diseases, no intervention study on depression and anxiety has been conducted in patients with CHD. The purpose of the present study is to explore the intervention effect of the dual-track interactive nursing intervention model on the depression and anxiety in elderly patients with CHD and its internal mechanism to provide empirical evidence for future intervention.

\section{SUBJECTS AND METHODS}

\section{Participants}

From 2018 to 2019,236 elderly patients with CHD (mean age $=63.5$ years) from three communities in Changsha, Hunan Province, China were selected as subjects. All the investigators were from the First Xiangya Hospital of Central South University, the Second Xiangya Hospital of Central South University, and the Third Xiangya Hospital of Central South University. The diagnostic criteria were as follows: (1) the patient met the diagnostic criteria for CHD developed by the World Health Organization and the International Society and Federation of Cardiology; (2) the patient was clearly diagnosed using various examinations, including physical examination, electrocardiogram (ECG), myocardial enzymogram, and coronary angiography. Based on the results, 103 patients with CHD, including 58 males and 50 females aged 42 78 years with an average age of 63.5 , were included in the special nursing intervention experimental study. The subjects who voluntarily participated in the intervention experiment were randomly divided using the random number table into the control (50 members) and the intervention (55 members) groups. Significant differences in gender, age structure, education level, and illness severity were not found between the two groups. Hence, the two groups were comparable.

All the participants in this experiment had already understood the purpose and method of the experiment before the experiment started and participated voluntarily. In addition, the experiment obtained the approval of the Ethics Committee of the Second Xiangya Hospital of Central South University.

\section{Methods}

The General Data Questionnaire, SF-36, SAS, and SDS were distributed by the full-time nursing staff designated by the research team to the 236 elderly patients with CHD enrolled in the hospital. The head was removed from all the scales, and the responsible nurses of the department instructed the patients, their family, and their physicians to participate in the questionnaire survey. (1) The subjective feelings towards the surveyed items within one week before admission were investigated, and the scales were collected for evaluation within the prescribed time. (2) The responsible nurses of the research team were familiar with the research process of the entire project. In addition, the use and evaluation methods of the scales were professionally trained by the teachers of the psychological teaching and research department of the school. (3) All the patients in this study had clear consciousness, no obstruction from their illness and physical condition, the ability to engage in effective language communication, and no barriers to understanding. Moreover, informed consent was obtained from all the patients.

The two groups of patients routinely used antiplatelet drugs, drugs to regulate blood lipids, drugs to dilate coronary artery, and other drugs to treat CHD.

The patients in the control group only used routine nursing measures, whereas the intervention group was subjected to special nursing intervention by the full-time nursing staff in the control group on the basis of routine nursing.

\section{Dual-track nursing intervention}

\section{Horizontal-track intervention}

The horizontal intervention track consisted of general practitioners and general nurses in the community where the patient lives, the patient, and the patient's family members. An illness intervention file was established for each patient, and the patient number, name, gender, and telephone number were marked. The file included various assessment forms for the patient: general condition checklist, health checklist, lifestyle and medication sheet, and health assessment form. The intervention lasted three months. The horizontal intervention track before the intervention covered ECG, cardiac ultrasound, routine laboratory examination of cardiology, myocardial isotope detection, coronary angiography, and multiconductivity physiology examination. In addition, the horizontal intervention track was recorded in a health checklist to establish an electronic file. A healthcare contact card with contact details of the corresponding healthcare provider was issued to each patient in duplicate. Given the community health service center working as the main body, a community health education lecture, dominated by the medical staff of this study, was provided once a month to patients with CHD, family members of patients, and caregivers. The content of the lecture included changing the sittingbased lifestyle. The dangers of sitting-based lifestyle 
were introduced to guide the patients to establish the concept of reasonable exercise. The exercise should follow the " $1,3,5$, and 7 programs." In particular, the patient should exercise at least once a day for $30 \mathrm{~min}$ and at least 5 days a week, and the heart rate after exercise should not exceed 170-times. The lecture also included improving the dietary structure. The relationship between diet and cardiovascular diseases was explained. The specialist medical staff should formulate low-fat and low-salt diet for the patient, keep abreast of the patient's diet, correct unreasonable dietary behaviors in time, and affirm a reasonable diet. Patients who failed to achieve satisfactory disease control and suffered severe conditions under horizontal-track intervention were transferred to longitudinal-track intervention.

\section{Longitudinal-track intervention}

The longitudinal track was composed of staff professionals in nursing cardiovascular diseases in highlevel hospitals, simplifying the medical treatment channel. Staff professionals in charge of treatment transfers were set up in the branch outpatient department of internal medicine to treat the patients transferred from the community. For patients who only need outpatient treatment, the medical treatment channel can be simplified. Moreover, hospitalized patients should be intervened. According to doctor's orders, specialist nurses should fill the intervention table, including diet therapy, exercise therapy, medication precautions, causes of CHD and nursing, prevention of complications, and other content, providing professional nursing intervention reference to the community-family rehabilitation after discharge from the hospital to ensure continuity of care.

\section{Dual-track interactive intervention}

The content of dual-track interaction included doubletrack and patient-patient interactions. The longitudinalhorizontal dual-track intervention staff jointly negotiated to determine the health intervention target according to each patient's specific situation; develop corresponding health education prescriptions; encourage patients and their family members to share successful experience; enable patients to learn scientific lifestyles, methods, techniques, and measures from one another; and help patients build self-care and confidence to cooperate actively in treatment. The dual-track interaction also included the interaction of experience in controlling diseases and healthcare information. Patients in the Tencent QQ group were constructed by the longitudinal track, the horizontal track, the patients, and their family members to share a successful experience.

\section{Measuring Tools}

\section{The Short Form-36 Health Survey (SF-36)}

It was created from Boston, USA Health Research Institute based on MOS SF (medical outcomes study short from) developed by Stewartse (1987). SF-36 scale contains 36 questions that evaluate 8 health-related dimensions of quality of life, which fall into the two categories including physical health and mental health. The scale is applicable to the assessment on quality of life for the general population. It is widely used in China and has good reliability and validity.

\section{The Symptom Checklist 90 (SCL-90)}

The Symptom Checklist 90 (SCL-90) consisted of 90 items (Derogatis et al. 1973, Liu \& Zhang 2004), including nine factors (somatization, obsessive-compulsive symptoms, interpersonal sensitivity, depression, anxiety, hostility, paranoia, and psychosis), and used 5level scoring (1-5). The total score and score in each factor were used to understand the distribution characteristics of various mental symptoms.

\section{The Self-rating Anxiety Scale (SAS)}

The Self-rating Anxiety Scale (SAS) is a selfassessment tool for understanding anxiety in counseling settings (Zung 1971). According to the norms for the Chinese population, the cutoff standard SAS score is 50 points, with 50-59 points representing mild anxiety, 6069 points representing moderate anxiety, and $>70$ points representing severe anxiety.

\section{The Self-rating Depression Scale (SDS)}

The Self-rating Depression Scale (SDS) is a selfrating scale (Zung 1965). SDS has been widely applied by Chinese scholars for special populations, such as mental health patients, senior high school students, undergraduate students, and middle school teachers. Reliable results have been obtained (Zung 1965). In addition, the scale consisted of 20 declarative sentences, each item representing a relevant symptom. The 20 items covered four groups of specific symptoms of depression, namely, spirituality-emotionality and corporality, psychomotor, and psychological disorders. Each item was rated on a scale from 1 to $4: 1$ (never or occasionally), 2 (sometimes), 3 (frequently), 4 (always). The depression severity index was calculated using the equation: Depression severity index $=$ cumulative score of all items $/ 80$ (the highest total score). Score ranging from $0.25-1.0$ may be obtained. A score of 0.5 or below indicates no depression, $0.5-0.59$ indicates mild depression, 0.6-0.69 indicates moderate depression, and 0.7 or above indicates severe depression (Zung 1965).

\section{Laboratory examinations}

Laboratory examinations included the following ECG, cardiac ultrasound, routine laboratory examination of cardiology, myocardial isotope detection, coronary angiography, and multiconductivity physiology examination.

\section{Statistical Analysis}

The data were processed using the SPSS 17.0 statistical software. The quantitative data were expressed as mean \pm standard deviation. The means of the two groups were compared using the independent sample $t$ test. Intergroup comparison was conducted using the 
paired $t$ test. The qualitative data were expressed by the number of cases and the composition ratio. The chisquare test or the Wilcoxon rank-sum test was used for cross-group comparison. $\mathrm{P}<0.05$ indicated statistically significant difference.

\section{RESULTS}

\section{Comparison of general data between the intervention and the control groups}

As shown in Table 1, 53 members, including 29 males and 24 females, are identified in the intervention group, whereas 50 members, including 24 males and 26 females, are identified in the control group. No significant difference in gender, age, education level, free medical care, and cardiac function grading is found between the two groups, indicating that the baseline levels of the two groups are basically the same $(\mathrm{P}>0.05)$.
Moreover, no difference in general data is found between the two groups. However, the intervention experimental comparison of the two groups is statistically significant.

\section{Comparison of SF-36 between the intervention and the control groups before and after the 3-month intervention}

This is shown in Table 2.

\section{Comparison of changes in the ECG ST segment between the intervention and the control groups before and after 3-month intervention}

After three months of intervention, the total duration and the total is chemic load of the intervention group are significantly lower than those of the traditional longitudinal transfer group (Table 3).

Table 1. Comparison of baseline data between the intervention and the control groups

\begin{tabular}{lcccc}
\hline Item & $\begin{array}{c}\text { Experimental group } \\
(n=53)\end{array}$ & $\begin{array}{c}\text { Control group } \\
(n=50)\end{array}$ & $t / x^{2}$ & $P$ \\
\hline Age (years) & $61.96 \pm 4.29$ & $61.32 \pm 4.69$ & 0.73 & 0.47 \\
Gender (male/female) & $29 / 24$ & $24 / 26$ & 0.01 & 1.00 \\
Education level (primary school-university) & $14 / 15 / 22 / 2$ & $16 / 18 / 14 / 2$ & 0.01 & 1.00 \\
Family monthly income (thousand Yuan) & $2.52 \pm 1.17$ & $2.62 \pm 1.12$ & -0.41 & 0.68 \\
Cardiac function grading (II/III) & $29 / 24$ & $29 / 21$ & 0.03 & 0.98 \\
Caregiver (children/spouse/other) & $20 / 14 / 19$ & $20 / 17 / 13$ & 0.35 & 0.89 \\
Free medical care (Yes/No) & $18 / 35$ & $23 / 27$ & 1.32 & 0.58 \\
Course of coronary heart disease & $5.20 \pm 2.08$ & $4.80 \pm 2.00$ & 1.01 & 0.31 \\
\hline
\end{tabular}

Table 2. Comparison of scores in SF-36 dimensions between the intervention group and the control group before and after intervention

\begin{tabular}{lcccccc}
\hline \multirow{2}{*}{ Item } & \multicolumn{3}{c}{ Experimental group $(n=53)$} & \multicolumn{3}{c}{ Control group $(n=50)$} \\
& Before intervention & After intervention & $t$ & Before intervention & After intervention & $t$ \\
\hline PF & $4.16 \pm 1.39$ & $7.96 \pm 1.42$ & $-13.34^{\mathrm{a}}$ & $4.04 \pm 1.05$ & $6.32 \pm 2.10$ & $4.53^{\mathrm{b}}$ \\
RP & $4.15 \pm 1.35$ & $7.30 \pm 1.57$ & $-11.28^{\mathrm{a}}$ & $4.30 \pm 1.26$ & $5.70 \pm 1.94$ & $4.61^{\mathrm{b}}$ \\
BP & $3.01 \pm 1.40$ & $3.64 \pm 1.16$ & $-2.67^{\mathrm{a}}$ & $2.90 \pm 1.38$ & $2.82 \pm 1.49$ & $3.12^{\mathrm{b}}$ \\
VT & $2.96 \pm 1.51$ & $3.77 \pm 0.99$ & $-3.27^{\mathrm{a}}$ & $2.84 \pm 1.29$ & $2.90 \pm 1.41$ & $3.64^{\mathrm{b}}$ \\
SF & $4.15 \pm 1.45$ & $7.90 \pm 1.33$ & $-13.17^{\mathrm{a}}$ & $4.04 \pm 1.17$ & $6.00 \pm 2.11$ & $5.49^{\mathrm{b}}$ \\
RE & $2.62 \pm 1.21$ & $4.15 \pm 0.94$ & $-7.34^{\mathrm{a}}$ & $2.30 \pm 1.12$ & $3.40 \pm 1.42$ & $3.16^{\mathrm{b}}$ \\
MH & $4.15 \pm 1.11$ & $8.11 \pm 1.49$ & $-14.93^{\mathrm{a}}$ & $4.38 \pm 1.80$ & $5.48 \pm 2.13$ & $7.30^{\mathrm{b}}$ \\
GH & $1.96 \pm 2.15$ & $3.90 \pm 1.04$ & $-10.16^{\mathrm{a}}$ & $1.96 \pm 0.75$ & $2.58 \pm 1.38$ & $5.51^{\mathrm{b}}$ \\
\hline
\end{tabular}

Note: PF - physiological function; RP - role physical: BP - body pain; VT - vitality; SF - social function;

$\mathrm{RE}$ - role emotional; $\mathrm{MH}$ - mental health; GH - general health; ${ }^{\mathrm{a}} \mathrm{P}<0.01$ compared with the value during enrollment;

${ }^{\mathrm{b}} \mathrm{P}<0.05$ comparison between the two groups.

No significant difference in the SCL-90 score is found between the intervention and the control groups before the intervention, whereas significant differences in SF-36 dimension scores are found between the two groups after the intervention $(\mathrm{P}<0.05)$

Table 3. Changes in the ECG ST segment of the intervention and the control groups after intervention

\begin{tabular}{lccc}
\hline Group & ST segment depression times & $\begin{array}{c}\text { Total duration of ST } \\
\text { segment depression }\end{array}$ & Total Ischemic load \\
\hline Experimental group $(n=53)$ & $64.66 \pm 3.13$ & $53.24 \pm 4.54$ & $105.54 \pm 16.07$ \\
Control group $(n=50)$ & $84.42 \pm 3.52$ & $102.82 \pm 5.04$ & $127.26 \pm 48.56$ \\
$\mathrm{t}$ & $-29.75^{* *}$ & $-52.45^{* *}$ & $-3.08^{*}$ \\
\hline
\end{tabular}

\footnotetext{
* statistically evident difference: $\mathrm{P}<0.05 ; \quad * *$ significant difference: $\mathrm{P}<0.001$
} 


\section{Comparison of scores in anxiety between the intervention and the control groups before and after 3-month intervention}

As shown in Table 4, no significant difference in the SAS score is found between the intervention and the control groups before the intervention (both at a high level). However, the SAS score of the intervention group is significantly lower than that of the control group after special nursing intervention.

Table 4. Comparison of scores in anxiety between the intervention and the control groups before and after intervention

\begin{tabular}{lcc}
\hline Group & $\begin{array}{c}\text { Before } \\
\text { intervention }\end{array}$ & $\begin{array}{c}\text { After } \\
\text { intervention }\end{array}$ \\
\hline Experimental group $(n=53)$ & $2.99 \pm 0.18$ & $2.04 \pm 0.18$ \\
Control group $(n=50)$ & $3.00 \pm 0.21$ & $2.82 \pm 0.29$ \\
$\mathrm{t}$ & -0.14 & $-16.26^{* *}$ \\
\hline * statistically evident difference: $\mathrm{P}<0.05 ;$ & \\
** significant difference: $\mathrm{P}<0.001$ &
\end{tabular}

\section{Comparison of scores in depression between the intervention and the control groups before and after 3-month intervention}

As shown in Table 5, no significant difference in the SDS score is found between the intervention and the control groups before the intervention (both at a high level). However, the SDS score of the intervention group is significantly lower than that of the control group after special nursing intervention.

Table 5. Comparison of scores in depression between the intervention and the control groups before and after intervention

\begin{tabular}{lcc}
\hline Group & $\begin{array}{c}\text { Before } \\
\text { intervention }\end{array}$ & $\begin{array}{c}\text { After } \\
\text { intervention }\end{array}$ \\
\hline Experimental group $(n=53)$ & $2.99 \pm 0.20$ & $1.94 \pm 0.16$ \\
Control group $(n=50)$ & $3.02 \pm 0.20$ & $2.66 \pm 0.55$ \\
$\mathrm{t}$ & -0.76 & $-8.99 * *$ \\
\hline \multirow{2}{*}{$*$ significant difference: $\mathrm{P}<0.001$} &
\end{tabular}

Comparison of changes in quality of life between the intervention and the control groups before and after 3-month intervention

No significant difference in the scores of SCL-90 is found between the two groups before the intervention (Table 6). After the intervention, no significant change is found in the scores of the control group. However, significant decreases are observed in the intervention group, suggesting that the quality of life of the intervention group has improved. The most evident improvement can be seen in anxiety $(P<0.001)$, followed by hostility $(P<0.005)$. Moreover, the most insignificant difference can be seen in somatization $(P<0.03)$, followed by fear $(P<0.02)$.

\section{DISCUSSION}

Based on the results of the present study, no significant difference in the basic conditions, including gender, age, and course of the disease, is found between the intervention and the control groups, indicating that the baseline levels of the two groups are consistent. With regard to the physiological functions of patients with CHD, a significant improvement can be seen either in the routine longitudinal transfer group or in those who received the dual-track interactive nursing intervention. Even in the changes in ECG ST segment, the patients in the intervention group have better and healthier performance compared with those in the control group. This finding is consistent with the results of Liu et al. (2017) and suggests that the implementation of a certain intervention and guidance for patients with CHD restores the physiological functions of patients with CHD, highlighting the necessity to strengthen the prognostic intervention (Kuhlmann et al. 2019). For the intervention group, the physiological functions of patients with CHD are significantly improved after 1-year intervention in dualtrack interactive nursing intervention model compared with that in the preintervention level and the control group, indicating that the dual-track interactive nursing intervention model has a better effect on improving the physiological functions of patients with CHD.

Table 6. Comparison of scores in dimensions of quality of life between the intervention and the control groups before and after intervention

\begin{tabular}{|c|c|c|c|c|c|c|c|}
\hline \multirow[t]{2}{*}{ Item } & \multicolumn{3}{|c|}{ Intervention group $(n=53)$} & \multicolumn{3}{|c|}{ Control group $(n=50)$} & \multirow[b]{2}{*}{$P$} \\
\hline & $\begin{array}{c}\text { Before } \\
\text { intervention }\end{array}$ & $\begin{array}{c}\text { After } \\
\text { intervention }\end{array}$ & $t$ & $\begin{array}{c}\text { Before } \\
\text { intervention }\end{array}$ & $\begin{array}{c}\text { After } \\
\text { intervention }\end{array}$ & $t$ & \\
\hline Somatization & $1.99 \pm 0.22$ & $1.51 \pm 0.14$ & $14.15 * *$ & $2.01 \pm 0.22$ & $1.96 \pm 0.27$ & 1.08 & 0.03 \\
\hline Obsession & $2.01 \pm 0.23$ & $1.49 \pm 0.13$ & $14.47 * *$ & $2.01 \pm 0.24$ & $1.93 \pm 0.33$ & 1.40 & 0.009 \\
\hline Interpersonal relationship & $1.93 \pm 0.24$ & $1.48 \pm 0.16$ & $12.39 * *$ & $1.97 \pm 0.24$ & $1.99 \pm 0.23$ & -0.36 & 0.007 \\
\hline Depression & $2.03 \pm 0.22$ & $1.49 \pm 0.12$ & $15.90 * *$ & $2.01 \pm 0.22$ & $1.95 \pm 0.12$ & 1.28 & 0.01 \\
\hline Anxiety & $2.00 \pm 0.22$ & $1.47 \pm 0.16$ & $12.85 * *$ & $2.02 \pm 0.27$ & $1.93 \pm 0.16$ & 1.76 & 0.001 \\
\hline Hostility & $1.97 \pm 0.30$ & $1.54 \pm 0.19$ & $8.12 * *$ & $2.07 \pm 0.31$ & $1.93 \pm 0.37$ & 1.79 & 0.005 \\
\hline Terror & $2.04 \pm 0.28$ & $1.51 \pm 0.20$ & $11.16^{* *}$ & $2.02 \pm 0.30$ & $1.98 \pm 0.36$ & 0.64 & 0.02 \\
\hline Paranoia & $2.05 \pm 0.36$ & $1.47 \pm 0.21$ & $10.24 * *$ & $1.99 \pm 0.36$ & $1.89 \pm 0.21$ & 1.25 & 0.009 \\
\hline Psychosis & $1.95 \pm 0.27$ & $1.51 \pm 0.14$ & $11.07 * *$ & $2.04 \pm 0.27$ & $2.00 \pm 0.30$ & 0.66 & 0.008 \\
\hline
\end{tabular}


Improvement is evident particularly in the five dimensions, including physical function, role physical, social function, mental health, and general health. First, with regard to mental health, the scores of the elderly patients with CHD who received dual-track interactive nursing intervention are significantly improved. This finding indicates that the dual-track interactive nursing intervention model can effectively improve the positive emotional experience of the elderly patients with CHD, reduce their depression and other negative emotional experience, enhance their emotional control, and bring more positive psychological feelings (Welton et al. 2019). This intervention result also supports the positive effect of this model on reducing depression and anxiety in patients with CHD. Second, regarding social and physical functions, the dual-track interactive nursing intervention model can be more effective than routine longitudinal transfer intervention. On the one hand, the simultaneous improvement in social, physical, and physiological functions, reflects that physiological health is closely related to social function. On the other hand, this improvement reflects that positive changes in physiological health promote changes in individuals' social functions (Ski et al. 2016). These findings support the research result that physical exercise improves and promotes physical and mental functions to a certain extent (Kachur et al. 2017) and shows that only physical exercise intervention is far from being sufficient. A comprehensive dual-track interactive nursing intervention model is more effective than a single physical exercise. Finally, the improvement in general health is also evident, but the improvement is slightly smaller than the improvement in mental health and physiological function. This finding reveals that the consideration of the overall health of elderly patients with CHD should be combined with physical and mental health. In particular, attention to the mental and emotional health of the elderly with CHD is the key to improve overall health (Deckers et al. 2017). Concisely, the dual-track interactive nursing intervention model improves the physical functions of the elderly with CHD and helps manage their mental and emotional state, contributing to mental health.

Based on the results of the present study, no significant difference in the experience of depression and anxiety is found between the intervention and the control groups before the intervention. However, after the intervention, significant decreases can be seen in the depression and anxiety scores of the intervention group, where as no significant decrease can be seen in the control group. First, this finding is consistent with the results of Bai \& Yang (2016) who randomly divided a total of 108 patients with CHD accompanied by depression and anxiety into the intervention and the control groups. The control group is subjected to drug therapy and routine nursing, whereas the intervention group is subjected to special nursing intervention. After the intervention, the depression and anxiety of the intervention group are alleviated. Moreover, the hospitalization time is shortened, and the cure rate is improved. In their research, special nursing content includes psychological intervention, behavioral correction, lifestyle intervention, and family factor intervention, taking into account the joint influence of the patient, the patient's lifestyle, and external environmental factors. This intervention is the same as the dual-track interactive nursing intervention model in the present study. Both interventions emphasize the ability of self-management and self-recovery in patients with CHD (Palacios et al. 2017). Anxiety in patients with CHD is related to their death anxiety about rehabilitation and health due to the variability of CHD that likely cause death in a short period of time. These uncertainties further strengthen the death anxiety in elderly patients with CHD (Halvari et al. 2019). In the dual-track interactive nursing intervention model, the condition is conveyed to the patient on the one hand. In this way, the patient can realize that CHD is not terrible, but blind treatment and unhealthy lifestyle are truly terrible. On the other hand, exercise and psychological intervention are used to enhance the patient's sense of control over his or her own body. This intervention allows patients to realistically understand their own condition and improves their sense of control over their physical skills, thereby reducing their fear of CHD (Qin et al. 2019). According to the Terror Management Theory, people's fears come mostly from the failure to control. Hence, increasing the self-control of elderly people with CHD through intervention can effectively reduce their death fear (Qin et al. 2019). The selfdetermination theory also points out that the occurrence of physiological diseases is closely related to the failure to meet the basic psychological needs of the individual. In addition, the satisfaction of basic psychological needs in the treatment and intervention processes can help the individual recover from the disease (Slovinec et al. 2014). In the dual-track interactive nursing intervention model, necessary guidance and freedom are provided to patients, greatly satisfying patient's need for autonomy. Based on the targeted intervention plan established for patients, the elderly patients can satisfy the need for competence by completing the tasks. Finally, maintaining an intimate connection with others contributes to meeting patient's need for belongingness. Therefore, the dual-track interactive nursing intervention model, which respects psychological needs and highlights the inner needs of the elderly, is helpful in improving the mental health of elderly patients with CHD. Moreover, anxiety and depression are interrelated in the neural mechanism of occurrence and development. Therefore, the dual-track interactive nursing intervention model is conducive to reducing anxiety in elderly patients with CHD while reducing depression (Miller et al. 2015).

Based on the statistical results, after 1-year intervention, the quality of life of elderly patients with CHD in the intervention group is remarkably improved. By contrast, the improvement in quality of life of elderly patients with CHD in the routine longitudinal transfer intervention group does not reach a significant level. This finding is consistent with the result obtained by Sun (2018) who used psychological nursing combined 
with WeChat intervention to treat 160 elderly patients with CHD. In the present study, the dual-track interactive nursing intervention model emphasizes psychological intervention for patients. The consistent results indicate that conducting mental health intervention among elderly patients with CHD is necessary (Vaccarino et al. 2017). In the present study, SCL-90, which includes physical and mental health, is used to measure the overall quality of life of elderly patients with CHD and comprehensively reflects the positive effect of the dual-track interactive nursing intervention model on improving quality of life of elderly patients with CHD. Although routine longitudinal transfer intervention can help restore the physiological functions of elderly patients with $\mathrm{CHD}$ and reduce their negative emotional experiences, such as depression and anxiety, in a certain period of time (RahmatiNajarkolaei et al. 2015), the effect is not ideal in long term. The dual-track interactive nursing intervention model, which emphasizes the basic psychological needs of patients and comprehensively considers the whole ecosystem where the patient is, achieves better effect than routine intervention. The advantages are as follows. First, the dual-track interactive nursing intervention model enables elderly patients to take the initiative, fully meeting their basic psychological needs. Routine intervention and behavioral intervention either overemphasize drug therapy or overemphasize changes in patients' perception. On the contrary, the dual-track interactive nursing intervention model reverses the subject of intervention, fully respects the characteristics of the elderly, exerts subjective initiative, satisfies basic psychological needs, and starts from physiological health to promote mental health. Moreover, the dual-track interactive nursing intervention model comprehensively improves the physical and mental health of elderly patients with CHD (Dickens et al. 2013). Second, the intervention is carried out in all aspects of the ecosystem in which the elderly are located, taking into account various microsystems (the relationship between the family and the elderly) and macrosystems (treatment environment in hospitals). This type of intervention can provide the elderly with social support and psychological warmth, improve patient's negative emotional and psychological state, and enhance patient's self-efficacy against disease, thereby achieving a great synergetic effect (Calderon \& Bellinger 2015).

\section{CONCLUSION}

CHD is a common illness with a high incidence in elderly worldwide. Patients with CHD often suffer emotional disorders, featured by depression and anxiety, due to the particularity of chronic diseases. In this study, the dual-track interactive nursing intervention model is used to engage elderly patients with anxiety and depression through SCL-90, SAS, SDS, Quality of Life Questionnaire, and laboratory examinations. The results of the study show that after the implementation of dual-track interactive nursing intervention model, the somatization, obsessive-compulsive symptoms, interpersonal sensiti- vity, depression, anxiety, hostility, and paranoia of the intervention group are significantly improved. Correspondingly, positive response to the disease is significantly improved in the intervention group. Simultaneously, the heart function test of the intervention group suggests that the condition has improved. For elderly patients with CHD and negative emotions, such as depression and anxiety, a more targeted dual-track interactive nursing intervention model based on routine longitudinal transfer can effectively improve their physiological functions, such as physical and physiological functions, and effectively enhance their social function and mental health, thereby reducing negative emotions, like depression and anxiety, and comprehensively improving quality of life. The dualtrack interactive nursing intervention model has an important role in promoting the prognosis of elderly patients with CHD. This study provides a valuable objective basis for clinical treatment and nursing of chronic diseases in elderly and is worthy of clinical application.

\section{Acknowledgements: None.}

\section{Conflict of interest: None to declare.}

\section{Contribution of individual authors:}

Jiao Yang: conceptualization, methodology, formal analysis, investigation, data, writing - original draft preparation, review and editing.

Hongling Hu: investigation, writing - review and editing. Yamin Li: conceptualization, methodology.

All authors have read and agreed to the published version of the manuscript.

\section{References}

1. Bai CH, Yang CQ: Effect of comprehensive assessment nursing intervention for the elderly on the psychological status of patients with coronary heart disease in the elderly ward. Journal of Modern Medicine \& Health 2018; 34:2575-2577

2. Bonaccio M, Di Castelnuovo A, Costanzo S, Persichillo M, De Curtis A, Cerletti C, et al: Health-related quality of life and risk of composite coronary heart disease and cerebrovascular events in the Moli-sani study cohort. European journal of preventive cardiology 2018; 25:287-297

3. Calderon $J \&$ Bellinger DC: Executive function deficits in congenital heart disease: why is intervention important? Cardiology in the Young 2015; 25:1238-1246

4. Catalan-Serra P, Campos-Rodriguez F, Reyes-Nuñez N, Selma-Ferrer MJ, Navarro-Soriano C, Ballester-Canelles $M$, et al: Increased Incidence of Stroke, but Not Coronary Heart Disease, in Elderly Patients With Sleep Apnea: Role of Continuous Positive Airway Pressure Treatment. Stroke 2019; 50:491-494

5. Deckers K, Schievink SH, Rodriquez MM, van Oostenbrugge RJ, van Boxtel MP, Verhey FR, et al: Coronary heart disease and risk for cognitive impairment or dementia: Systematic review and meta-analysis. PLoS One 2017; 12:e0184244 
6. Derogatis LR, Lipman RS, Covi L: SCL-90: an outpatient psychiatric rating scale - preliminary report. Psychopharmacol Bull 1973; 9:13-28

7. Dickens C, Cherrington A, Adeyemi I, Roughley K, Bower $P$, Garrett $C$, et al: Characteristics of psychological interventions that improve depression in people with coronary heart disease: a systematic review and metaregression. Psychosomatic medicine 2013; 75:211-221

8. Halvari AEM, Halvari H, \& Deci EL: Dental anxiety, oral health-related quality of life, and general well-being: $A$ self-determination theory perspective. Journal of Applied Social Psychology 2019; 49:295-306

9. Kachur S, Chongthammakun V, Lavie CJ, De Schutter A, Arena $R$, Milani $R V$, et al: Impact of cardiac rehabilitation and exercise training programs in coronary heart disease. Progress in cardiovascular diseases 2017; 60:103-114

10. Khandaker GM, Zuber V, Rees JM, Carvalho L, Mason AM, Foley CN, et al: Shared mechanisms between coronary heart disease and depression: findings from a large UK general population-based cohort. Molecular psychiatry 2019; Published Online

11. Kuhlmann SL, Arolt V, Haverkamp W, Martus P, Ströhle A, Waltenberger J: Prevalence, 12-Month Prognosis, and Clinical Management Need of Depression in Coronary Heart Disease Patients: A Prospective Cohort Study. Psychotherapy and psychosomatics 2019; 88:300-311

12. Liu H, Zhang JX: Norm of Symptom Checklist (SCL-90) in Chinese Middle School Students. Chin Ment Health J 2004; 18:88-90

13. Liu RT, Hernandez EM, Trout ZM, Kleiman EM, \& Bozzay ML: Depression, social support, and long-term risk for coronary heart disease in a 13-year longitudinal epidemiological study. Psychiatry research 2017; 251:36-40

14. Miller BR, \& Hen R: The current state of the neurogenic theory of depression and anxiety. Current opinion in neurobiology 2015; 30:51-58

15. Palacios J, Lee GA, Duaso M, Clifton A, Norman IJ, Richards D, et al: Internet-delivered self-management support for improving coronary heart disease and selfmanagement-related outcomes: A systematic review. The Journal of cardiovascular nursing 2017; 32:E9

16. Qin L, Cui CH, Huo YL, Yang XC, \& Zhao YQ: Clinical Efficacy of Physical Factors Combined with Early Psychological Intervention in Treatment of Patients with Chron-ic Limb Pain. Iranian Journal of Public Health 2019; 48:858-863

17. Rahmati-Najarkolaei F, Ghaffarpasand E, Gholami Fesharaki M, Jonaidi-Jafari N: Nutrition and physical activity educational intervention on CHD risk factors: a systematic review study. Archives of Iranian medicine $2015 ; 18: 51-57$

18. Richards SH, Anderson L, Jenkinson CE, Whalley B, Rees $K$, Davies $P$, et al: Psychological interventions for coronary heart disease: Cochrane systematic review and meta-analysis. European Journal of Preventive Cardiology 2018; 25:247-259
19. Schuch FB, Vasconcelos-Moreno MP, Borowsky C, Zimmermann AB, Rocha NS \& Fleck MP: Exercise and severe major depression: effect on symptom severity and quality of life at discharge in an inpatient cohort. Journal of psychiatric research 2015; 61:25-32

20. Ski CF, Jelinek M, Jackson AC, Murphy BM, \& Thompson DR: Psychosocial interventions for patients with coronary heart disease and depression: A systematic review and meta-analysis. European Journal of Cardiovascular Nursing 2016; 15:305-316

21. Slovinec D'Angelo ME, Pelletier LG, Reid RD \& Huta V: The roles of self-efficacy and motivation in the prediction of short-and long-term adherence to exercise among patients with coronary heart disease. Health Psychology 2014; 33:1344

22. Stewart AL, Hays RD, Ware JE: The MOS 36-item short form health survey (SF-36): Conceptual framework and item selection. Medical Care 1987; 26:724-35

23. Sun HM: Effect of WeChat follow-up nursing on contraceptive self-efficacy and self-care ability in patients with painless abortion. Today Nurse 2018; 25:97-100

24. Vaccarino $V$ \& Bremner JD: Behavioral, emotional and neurobiological determinants of coronary heart disease risk in women. Neuroscience \& Biobehavioral Reviews 2017; 74:297-309

25. Valbusa F, Agnoletti D, Scala L, Grillo C, Arduini P, Bonapace $S$, et al: Non-alcoholic fatty liver disease and increased risk of all-cause mortality in elderly patients admitted for acute heart failure. International journal of cardiology 2018; 265:162-168

26. Vermunt NP, Harmsen M, Westert GP, Rikkert MGO, \& Faber MJ: Collaborative goal setting with elderly patients with chronic disease or multimorbidity: a systematic review. BMC geriatrics 2017; 17:167

27. Wagner M, Gelbrich G, Kircher J, Kotseva K, Wood D, Morbach $C$, et al: Secondary prevention in younger vs. older coronary heart disease patients - Insights from the German subset of the EUROASPIRE IV survey. International journal of behavioral medicine 2018; 25:283-293

28. Welton NJ, Caldwell DM, Adamopoulos E, Vedhara K: Mixed treatment comparison meta-analysis of complex interventions: psychological interventions in coronary heart disease. American Journal of Epidemiology 2009; 169:1158-1165

29. Yin $P$, Ma Q, Wang L, Lin P, Zhang M, Qi $S \&$ Wang Z: Chronic obstructive pulmonary disease and cognitive impairment in the Chinese elderly population: a large national survey. International journal of chronic obstructive pulmonary disease 2016; 11:399

30. Zhang $Y$, Chen $Y$ \& Ma L: Depression and cardiovascular disease in elderly: Current understanding. Journal of Clinical Neuroscience 2018; 47:1-5

31. Zung $W W:$ A self-rating depression scale. Archives of general psychiatry 1965; 12:63-70

32. Zung WW: A rating instrument for anxieties orders. Psychosomatics 1971; 12:371-379

Correspondence:

Yamin Li, MD

The Second Xiangya Hospital, Central South University Changsha, Hunan, 410011, China

E-mail:liyamin3336@163.com 